\title{
New Approach in Fixed Resource Allocation and Target Setting Using Data Envelopment Analysis with Common Set of Weights
}

\author{
Marzieh Ghasemi, ${ }^{1}$ Mohammad Reza Mozaffari $\mathbb{D}^{2},{ }^{2}$ Farhad Hosseinzadeh Lotfi, ${ }^{1}$ \\ Mohsen Rostamy malkhalifeh, ${ }^{1}$ and Mohammad Hasan Behzadi ${ }^{3}$ \\ ${ }^{1}$ Department of Mathematics, Science and Research Branch, Islamic Azad University, Tehran, Iran \\ ${ }^{2}$ Department of Mathematics, Shiraz Branch, Islamic Azad University, Shiraz, Iran \\ ${ }^{3}$ Department of Statistics, Science and Research Branch, Islamic Azad University, Tehran, Iran
}

Correspondence should be addressed to Mohammad Reza Mozaffari; mozaffari854@yahoo.com

Received 2 July 2021; Revised 2 September 2021; Accepted 10 December 2021; Published 12 January 2022

Academic Editor: Jorge-Antonio Lopez-Renteria

Copyright (C) 2022 Marzieh Ghasemi et al. This is an open access article distributed under the Creative Commons Attribution License, which permits unrestricted use, distribution, and reproduction in any medium, provided the original work is properly cited.

One of the mathematical programming techniques is data envelopment analysis (DEA), which is used for evaluating the efficiency of a set of similar decision-making units (DMUs). Fixed resource allocation and target setting with the help of DEA is a subject that has gained much attention from researchers. A new model was proposed by determining a common set of weights (CSW). All DMUs were involved with the aim of achieving higher efficiency in every DMU after the procedure. The minimum resources and targets allocated to each DMU were commensurate to the efficiency of that DMU and the share of DMU in the input resources and the output productions. To examine the proposed method, other methods in the DEA literature were examined as well, and then, the efficiency of the method was demonstrated through a numerical example.

\section{Introduction}

The original concepts related to DEA were first introduced in 1978 by Charnes et al. [1] and rapidly gained popularity among researchers. By introducing the CCR model, Charnes was able to present a novel method for efficiency measurement in units. A topic that has been of interest to researchers is fixed resource allocation and target setting using DEA.

To reduce the costs, the subsidiaries of an organization are forced to cooperate with each other. The costs allocated to the infrastructure are called fixed costs. Another issue that can be discussed in an organization is the method used for fixed resource allocation and target setting. Let us assume that a given organization has limited resources and wants to allocate those resources to its subsidiaries and set targets for them based on their performance. Now, the questions would be how to allocate the resources to the subsidiaries in an equitable manner and how to carry out the target setting.

In 1999, Cook and Cross [2] introduced the two axioms of invariance-efficiency and Pareto-minimality and studied the input in the fixed resource allocation problem. In the method proposed by them based on these two axioms, only the equitability of the resource allocation was taken into consideration.

The method provided by Cook and Kress [2] was generalized from input-oriented to output-oriented conditions and from CCR to BCC model by Cook and Zhu [3]. Lin [4] proved that no optimal solution would be obtained by adding certain constraints to the method of Cook and Zhu [3]. Lin [4] added some specified purposes to their model to improve this situation and achieve the possible allocation.

In 2003, Beasly [5] presented a nonlinear method that maximized the average efficiency of all DMUs. Amirteimoori and Kordrostami [6] indicated that Beasley's method is not possible in some cases. Accordingly, they introduced a new DEA-based method for targeting and also allocating the fixed resources. In 2017, Jahanshahloo [7] et al. used the concept of CSW and the efficiency invariance axiom to present another method for fixed resource allocation. Furthermore, Jahanshahloo et al. [7] demonstrated that Beasley's [5] method, which was claimed impossible by Amirteimoori and Kordrostami [6], is always possible. Li et al. [8] extended the work carried out by Jahanshahloo et al. [7]. 
In 2004, using primary and dual problems, Jahanshahloo et al. [9] presented a formula that determined the fixed cost for each DMU without the need to solve any linear problems. In the meantime, Li et al. [10] viewed the problem of fixed resource allocation from a different perspective. They assumed that if there are any other costs as well, the allocated fixed costs shall then be added to these costs. Furthermore, in 2014, Du [11] carried out resource allocation using the concept of cross efficiency.

Hosseinzadeh et al. [12] introduced a model using invariance-efficiency principle where each DMU determines the minimum and maximum value that can participate in receiving fixed resources. Then, the convex combination of these values is considered as the fixed allocated resources. The resource allocation was conducted by Asgharinia et al. [13] using CSW. Amirteimoori et al. [14] and Hosseinzadeh et al. [15] studied resource allocation and targeting with the CSW.

In the CSW method, the ratio of weighted outputs to weighted inputs is simultaneously maximized for all DMUs. Thompson et al. [16,17], Cook et al. [18], Charnes et al. [19], and Roll et al. [20] have studied some of the CSW concepts. Jahanshahloo et al. [21, 22] introduced a multiobjective model for efficiency measurement through the CSW method in one study. Moreover, in another study, using the infinity norm, they proposed a nonlinear method for solving the CSW model. Furthermore, Cook and Zhu [23] utilized goal programming to solve the CSW model, and Davoodi and Rezai [24] suggested a linear method for the same purpose. Kachouei et al. [25] used CSW to efficiency evaluation in DEA with undesirable outputs when data is fuzzy.

The technique proposed in the current manuscript considers certain conditions that force the participation of all DMUs in the resource allocation and target setting. Imagine that a company wants to provide some resources to its subsidiaries in order for them to produce new products, while the amount of resources allocated to each subsidiary is to be in accordance with the performance of that subsidiary and its inputs relative to the inputs of the other subsidiaries [26-28]. Moreover, the company intends to set a target for each subsidiary based on its efficiency and its outputs relative to the outputs of the other subsidiaries [29-31]. In this manuscript, we consider the fixed resources as an additional input and the target setting as an output, and then using a CSW, we present a model that, while including all DMUs in the resource allocation and target setting, ensures that, after the procedure, the efficiency of all DMUs would be the same as before or higher.

Section 2 is devoted to basic models used in this paper. Section 3 describes the novel method proposed in this paper. In Section 4, the novel method is applied to a numerical example. And finally, Section 5 is dedicated to the conclusions and recommendations.

\section{Background}

This segment describes CCR model. Then, we present the CSW model.

2.1. CCR Model. Assume that $x_{i j} \quad(i=1, \ldots, m)$ and $y_{r j} \quad(r=1, \ldots, s)$ are the inputs and outputs consumed and produced by $\mathrm{DMU}_{j} \quad(j=1, \ldots, n)$, respectively. Also, let $v$ be the weight related to the inputs and $u$ the weight related to the outputs. CCR model [1] is as follows:

$$
\begin{aligned}
\theta_{o}^{*} & =\max \sum_{r=1}^{s} u_{r} y_{r o}, \\
\text { s.t }: \sum_{i=1}^{m} v_{i} x_{i o} & =1, \\
\sum_{r=1}^{s} u_{r} y_{r j}-\sum_{i=1}^{m} v_{i} x_{i j} & \leq 0, \quad j=1, \ldots, n \\
u_{r}, v_{i} & \geq 0, \quad r=1, \ldots, s i=1, \ldots, m .
\end{aligned}
$$

Definition 1. (CCR efficiency).

$\mathrm{DMU}_{o}$ is CCR-efficient if $\theta_{o}^{*}=1$ and there exists at least one optimal $\left(u^{*}, v^{*}\right)$ with $u^{*}>0$ and $v^{*}>0$. Otherwise, $\mathrm{DMU}_{o}$ is CCR-inefficient.

2.2. Common Set of Weights (CSW) Model. Model 1 must be solved $n$ times to assess $n$ DMUs. Model 1 is assessed through the best weights. To resolve this problem and obtain just a CSW for all DMUs and evaluate all DMUs based on a CSW, the CSW model can be used as follows [22]:

$$
\begin{cases}\operatorname{Max} \quad & \left\{\frac{\sum_{r=1}^{s} u_{r} y_{r 1}}{\sum_{i=1}^{m} v_{i} x_{i 1}}, \frac{\sum_{r=1}^{s} u_{r} y_{r 2}}{\sum_{i=1}^{m} v_{i} x_{i 2}}, \ldots, \frac{\sum_{r=1}^{s} u_{r} y_{r n}}{\sum_{i=1}^{m} v_{i} x_{i n}}\right\}, \\ \text { s.t: } \quad & \frac{\sum_{r=1}^{s} u_{r} y_{r j}}{\sum_{i=1}^{m} v_{i} x_{i j}} \leq 1, \quad i=1, \ldots, n, \\ & u_{r}, v_{i} \geq \varepsilon, \quad i=1, \ldots, n .\end{cases}
$$

Different methods have been proposed for solving this multiobjective fractional model (2). Goal programming (GP) is one of these methods [32-35]. In goal programming, the decision maker specifies certain levels for achieving the objectives. Moreover, the decision maker allows deviation from the goals and, therefore, creates flexibility in the decisionmaking process. Also, the objective function seeks to minimize the undesirable deviations. Based on the GP method, model (2) can be transformed into the following nonlinear model in order to identify a set of common weights:

$$
\begin{cases}\text { Min } \quad & \sum_{j=1}^{n}\left(s_{j}^{-}+s_{j}^{+}\right), \\ & \frac{\sum_{r=1}^{s} u_{r} y_{r j}}{\sum_{i=1}^{m} v_{i} x_{i j}}+s_{j}^{-}-s_{j}^{+}=A_{j}, \quad z=1, \ldots, n, \\ s . t: \quad & \frac{\sum_{r=1}^{s} u_{r} y_{r j}}{\sum_{i=1}^{m} v_{i} x_{i j}} \leq 1 \quad j=1, \ldots, n, \\ & s_{j}^{-}, s_{j}^{+} \geq 0, u_{r}, v_{i} \geq \varepsilon, \quad j=1, \ldots, n, i=1, \ldots, m, \mathrm{r}=1, \ldots, s .\end{cases}
$$


where $A_{j}$ denotes the goal of the $j$ th objective. $s_{j}^{-}$and $s_{j}^{+}$are negative and positive deviations from the objective, respectively. Technically, the deviational variables $s_{j}^{-}$and $s_{j}^{+}$ help the objective function $j$ to achieve the goal $A_{j}=1$. Thereby, the positive deviation is equal to zero here, i.e., $s_{j}^{+}=0$. Thus, the first constraint can be reformulated as follows:

$$
\sum_{r=1}^{s} u_{r} y_{r j}+s_{j}^{-} \sum_{i=1}^{m} v_{i} x_{i j}=\sum_{i=1}^{m} v_{i} x_{i j}, \quad j=1, \ldots, n .
$$

Considering the nonlinear constraint above, model (3) cannot be transformed into a linear model. In order to achieve the goal of $\mathrm{DMU}_{j}$ (efficiency scores of one), the numerator should increase in the fraction $\sum_{r=1}^{s} u_{r} y_{r j} / \sum_{i=1}^{m} v_{i} x_{i j}$ while the denominator is decreased. Therefore, model (3) can be reformulated as follows:

$$
\left\{\begin{array}{c}
\frac{\sum_{r=1}^{s} u_{r} y_{r j}+s_{j}^{+}}{\sum_{i=1}^{m} v_{i} x_{i j}-s_{j}^{-}}=1, \quad j=1, \ldots, n, \\
\operatorname{Min} \sum_{j=1}^{n}\left(s_{j}^{-}+s_{j}^{+}\right), s . t: \frac{\sum_{r=1}^{s} u_{r} y_{r j}}{\sum_{i=1}^{m} v_{i} x_{i j}} \leq 1, \quad j=1, \ldots, n, \\
s_{j}^{-}, s_{j}^{+} \geq 0, u_{r}, v_{i} \geq \varepsilon, \quad j=1, \ldots, n, i=1, \ldots, m, \mathrm{r}=1, \ldots, s .
\end{array}\right.
$$

Obviously, based on the first constraint of model (4), the second constraint is redundant and can be removed from the model. Therefore, the model can be formulated in the following linear form:

$$
\begin{cases}\text { Min } \quad & \sum_{j=1}^{n}\left(s_{j}^{-}+s_{j}^{+}\right), \\ & \sum_{r=1}^{s} u_{r} y_{r j}-\sum_{i=1}^{m} v_{i} x_{i j}+\left(s_{j}^{+}+s_{j}^{-}\right)=0, \quad j=1, \ldots, n, \\ & s_{j}^{-}, s_{j}^{+} \geq 0, u_{r}, v_{i} \geq \varepsilon \quad j=1, \ldots, n, i=1, \ldots, m, \mathrm{r}=1, \ldots, s .\end{cases}
$$

By setting $s_{j}^{-}+s_{j}^{+}=s_{j}$, the model is transformed as follows:

$$
\begin{cases}\text { Min } \quad & \sum_{j=1}^{n} S_{j}, \\ & \sum_{r=1}^{s} u_{r} y_{r j}-\sum_{i=1}^{m} v_{i} x_{i j}+\left(S_{j}\right)=0, \quad j=1, \ldots, n, \\ & S_{j} \geq 0, u_{r}, v_{i} \geq \varepsilon \quad j=1, \ldots, n, i=1, \ldots, m, \mathrm{r}=1, \ldots, s .\end{cases}
$$

If we let $\left(u_{r}^{*}, v_{i}^{*}, s_{j}^{*}\right)$ be the optimal solution of model (6), the CSW-efficiency of $\mathrm{DMU}_{j}$ is calculated as follows:

$$
\begin{aligned}
\theta_{j}^{\mathrm{csw}} & =\frac{\sum_{r=1}^{s} u_{r}^{*} y_{r j}}{\sum_{i=1}^{m} v_{r}^{*} y_{i j}} \\
& =1-\frac{s_{j}^{*}}{\sum_{i=1}^{m} v_{i}^{*} y_{i j}}, \quad j=1, \ldots, n .
\end{aligned}
$$

Definition 2. If $\theta_{O}^{\mathrm{csw} *}=1$, then $\mathrm{DMU}_{O}$ is efficient with the CSW resulting from model 2 and otherwise $\mathrm{DMU}_{O}$ is inefficient of common weights.

\section{Proposed Model}

Assume that $x_{i j} \quad(i=1, \ldots, m)$ and $y_{r j} \quad(r=1, \ldots, s)$ are the inputs and outputs consumed and produced by $\mathrm{DMU}_{j} \quad(j=1, \ldots, n)$, respectively. Now, consider a fixed resource $F$ and a general target $G$ that we want to equitably allocate to the DMUs. We consider $f_{j} \quad(j=1, \ldots, n)$ as the variable related to fixed resources and $g_{j} \quad(j=1, \ldots, n)$ as the variable related to target setting in $D M U_{j}$. It is obvious that

$$
\begin{aligned}
& \sum_{j=1}^{n} f_{j}=F, \\
& \sum_{j=1}^{n} g_{j}=G .
\end{aligned}
$$

The efficiency score of $\mathrm{DMU}_{j}$ when the resources are not allocated and the target is not set is represented by $\theta_{j}^{*}$, which is calculated using the multiplier CCR model. We consider the fixed resource as an additional input with the weight $v_{m+1}$, and the target corresponding to $\mathrm{DMU}_{j}$ as a new output with the weight $u_{s+1}$. In our approach, we carry out the fixed resource allocation and target setting in a way that the efficiency of each DMU after the procedure would be higher than or the same as the previous efficiency. That is:

$$
\theta_{j}^{*} \leq \frac{\sum_{r=1}^{s} u_{r} y_{r j}+u_{s+1} g_{j}}{\sum_{i=1}^{m} v_{i} x_{i j}+v_{m+1} f, \quad j=1, \ldots, n} .
$$


We also intend to achieve maximum efficiency through a CSW. Therefore, our objective is

$\max \left\{\frac{\sum_{r=1}^{s} u_{r} y_{r 1}+u_{s+1} g_{1}}{\sum_{i=1}^{m} v_{i} x_{i 1}+v_{m+1} f_{1}}, \ldots, \frac{\sum_{r=1}^{s} u_{r} y_{r m}+u_{s+1} g_{n}}{\sum_{i=1}^{m} v_{i} x_{i n}+v_{m+1} f_{n}}\right\}$.

We believe that all DMUs must be involved in the resource allocation and target setting. Therefore, for each DMU, we consider the least share in the allocated resources and the set targets. If the share that each DMU has in the inputs is multiplied by the efficiency of that DMU and the amount of fixed resources, the resulting value can be considered as a lower bound for the amount of fixed resources that DMU should receive. Also, if the share that each DMU has in the produced outputs is multiplied by the efficiency of that DMU and the overall target, the obtained value can be considered as a lower bound for target setting. To achieve our goals, we proceed as follows:

If $\lambda_{j}=1 / m \sum_{i=1}^{m} x_{i j} / \sum_{t=1}^{n} x_{i t}$ and $\mu_{j}=1 / s \sum_{r=1}^{m} \quad y_{r j} /$ $\sum_{t=1}^{n} y_{r t}$ are the shares of DMU $\mathrm{DM}_{j}$ in the input resources and the produced outputs, respectively, and $\theta_{j}^{*}$ stands for the efficiency of $\mathrm{DMU}_{j}$ prior to the allocation of fixed resources and target setting, which is obtained using the multiplier CCR model, then the minimum resources for $\mathrm{DMU}_{j}$ would be equal to $\theta_{j}^{*} \lambda_{j} F$, and the minimum target set for $\mathrm{DMU}_{j}$ would equal $\theta_{j}^{*} \mu_{j} G$. This means that the minimum fixed resources that each DMU would receive equals the amount of inputs it has consumed multiplied by its efficiency in achieving those inputs. Furthermore, the minimum target set for each DMU is equal to its outputs and the efficiency that it has had before fixed resource allocation. Therefore, any DMU that has a larger share in the inputs and can achieve higher efficiency with those inputs needs to receive more resources. And similarly, a higher target is set for any DMU that has higher efficiency and a larger contribution in the output generation. Therefore, the general model is formulated as follows:

$$
\begin{gathered}
\max \quad\left\{\frac{\sum_{r=1}^{s} u_{r} y_{r 1}+u_{s+1} g_{1}}{\sum_{i=1}^{m} v_{i} x_{i 1}+v_{m+1} f_{1}}, \ldots, \frac{\sum_{r=1}^{s} u_{r} y_{r m}+u_{s+1} g_{n}}{\sum_{i=1}^{m} v_{i} x_{i n}+v_{m+1} f_{n}}\right\}, \\
\text { s.t: } \theta_{j}^{*} \leq \frac{\sum_{r=1}^{s} u_{r} y_{r j}+u_{s+1} g_{j}}{\sum_{i=1}^{m} v_{i} x_{i j}+v_{m+1} f_{j}} \leq 1, \quad j=1, \ldots, n, \\
f_{j} \geq \theta_{j}^{*} \lambda_{j} F \leq \quad j=1, \ldots, n, \quad(12 \mathrm{a}) \\
g_{j} \geq \theta_{j}^{*} \mu_{j} G, \quad j=1, \ldots, n, \\
\sum_{j=1}^{n} f_{j}=F, \quad j=1, \ldots, n, \\
\sum_{j=1}^{n} g_{j}=G, \quad j=1, \ldots, n, \\
f_{j}, g_{j}, u_{r}, v_{i}, u_{s+1}, v_{m+1} \geq \varepsilon, \\
j=1, \ldots, n, r=1, \ldots, s, i=1, \ldots, m .
\end{gathered}
$$

The constraint (12a) assures that the efficiency of each DMU after allocating the resources and targeting is more than its efficiency before allocating the resources and targeting. The constraint (12b) represents the minimum amount allocated to the DMU. The constraint (12c) ensures the participation of all DMUs in targeting. The constraints (12d) and (12e) indicate that the total resources received and the total objective designated are equal to the amount of resources and the intended purpose of the decision maker, respectively.

Theorem 1. Model (10) is bounded.

Proof. Suppose that $\left(\bar{f}_{j}, \bar{g}_{j}, \bar{u}_{r}, \bar{v}_{i}, \bar{u}_{s+1}, \bar{v}_{m+1}\right)$ is a feasible solution for model (10). Therefore, this answer applies to all constraints of the matter. Hence, per $j$, we have

$$
\frac{\sum_{r=1}^{s} \bar{u}_{r} y_{r j}+\bar{u}_{s+1} \bar{g}_{j}}{\sum_{i=1}^{m} \bar{v}_{i} x_{i j}+\bar{v}_{m+1} \bar{f}_{j}} \leq 1 \text {. }
$$

This constraint represents that all objective functions of the fractional multiobjective model (10) are less than or equal to 1 . On the other hand, since the objective function is in the form of maximizing, the maximum possible value of the objective function is less than or equal to 1 . Then, the model is bounded.

Model 10 is a nonlinear multiobjective model. We convert this model into a single-objective linear model. By setting $v_{m+1} f_{j}=f_{j}^{\prime}$ and $u_{s+1} g_{j}=g_{j}^{\prime}$, model (10) would change as follows:

$$
\left\{\begin{array}{l}
\max \left\{\frac{\sum_{r=1}^{s} u_{r} y_{r 1}+g_{1}^{\prime}}{\sum_{i=1}^{m} v_{i} x_{i 1}+f_{1}^{\prime}}, \ldots, \frac{\sum_{r=1}^{s} u_{r} y_{r n}+g_{n}^{\prime}}{\sum_{i=1}^{m} v_{i} x_{i n}+f_{n}^{\prime}}\right\}, \\
\theta_{j}^{*} \leq \frac{\sum_{r=1}^{s} u_{r} y_{r j}+g_{1}^{\prime}}{\sum_{i=1}^{m} v_{i} x_{i j}+f_{j}^{\prime}} \leq 1, \quad j=1, \ldots, n, \\
f_{j}^{\prime} \geq v_{m+1} \theta_{j}^{*} \lambda_{j} F, \quad j=1, \ldots, n, \\
g_{j}^{\prime} \geq u_{s+1} \theta_{j}^{*} \mu_{j} G, \quad j=1, \ldots, n, \\
\sum_{j=1}^{n} f_{j}^{\prime}=v_{m+1} F, \quad j=1, \ldots, n, \\
j . t: \quad j, \ldots, n, r=1, \ldots, s, i=1, \ldots, m . \\
\sum_{j=1}^{n} g_{j}^{\prime}=u_{s+1}^{\prime} G, \quad j=1, \ldots, n,
\end{array}\right.
$$


Now, we change the multiobjective model into a linear single-objective model that can help us achieve our goals. To maximize the objective function, all we need to do is to maximize the numerator and minimize the denominator. As such, the proposed model can be rewritten as

$$
\begin{cases}\max & \left\{\sum_{r=1}^{s} u_{r} y_{r 1}+g_{1}^{\prime}-\sum_{i=1}^{m} v_{i} x_{i 1}-f_{1}^{\prime}, \ldots, \sum_{r=1}^{s} u_{r} y_{r n}+g_{n}^{\prime}-\sum_{i=1}^{m} v_{i} x_{i n}-f_{n}^{\prime}\right\} \\ & \sum_{r=1}^{s} u_{r} y_{r j}+g_{j}^{\prime}-\sum_{i=1}^{m} v_{i} x_{i j}-f_{j}^{\prime} \leq 0, \quad j=1, \ldots, n, \\ & s . t: \sum_{r=1}^{s} u_{r} y_{r j}+g_{j}^{\prime}-\theta_{j}^{*} \sum_{i=1}^{m} v_{i} x_{i j}-\theta_{j}^{*} f_{j}^{\prime} \geq 0, \quad j=1, \ldots, n, \\ & f_{j}^{\prime} \geq v_{m+1} \theta_{j}^{*} \lambda_{j} F, \quad j=1, \ldots, n, \\ & g_{j}^{\prime} \geq u_{s+1} \theta_{j}^{*} \mu_{j} G, \quad j=1, \ldots, n, \\ & \sum_{j=1}^{n} f_{j}^{\prime}=v_{m+1} F, \quad j=1, \ldots, n, \\ & \sum_{j=1}^{n} g_{j}^{\prime}=u_{s+1} G, \quad j=1, \ldots, n, \\ & f_{j}^{\prime}, g_{j}^{\prime}, u_{r}, v_{i}, u_{s+1}, v_{m+1} \geq \varepsilon, \quad i=1, \ldots, m, r=1, \ldots, s, j=1, \ldots, n .\end{cases}
$$

By converting the first constraint into an equation and changing the Max to Min, model (15) would change as follows:

$$
\begin{cases}\min \quad & \left\{S_{1}, \ldots, S_{n}\right\}, \\ & \sum_{r=1}^{s} u_{r} y_{r j}+g_{j}^{\prime}-\sum_{i=1}^{m} v_{i} x_{i j}-f_{j}^{\prime}+S_{j}=0, \quad j=1, \ldots, n, \\ & s . t: \sum_{r=1}^{s} u_{r} y_{r j}+g_{j}^{\prime}-\theta_{j}^{*} \sum_{i=1}^{m} v_{i} x_{i j}-\theta_{j}^{*} f_{j}^{\prime} \geq 0, \quad j=1, \ldots, n, \\ & f_{j}^{\prime} \geq v_{m+1} \theta_{j}^{*} \lambda_{j} F, \quad j=1, \ldots, n, \\ & g_{j}^{\prime} \geq u_{s+1} \theta_{j}^{*} \mu_{j} G \quad j=1, \ldots, n, \\ & \sum_{j=1}^{n} f_{j}^{\prime}=v_{m+1} F, \quad j=1, \ldots, n, \\ & \sum_{j=1}^{n} g_{j}^{\prime}=u_{s+1} G, \quad j=1, \ldots, n, \\ & f_{j}^{\prime}, g_{j}^{\prime}, S_{j} \geq 0, u_{r}, v_{i}, u_{s+1}, v_{m+1} \geq \varepsilon, \quad j=1, \ldots, n, \quad r=1, \ldots, s, \quad i=1, \ldots, m .\end{cases}
$$

Model (16) is a multiobjective model whose goal is to minimize the covariates $S_{j}$. We convert the multiobjective model to the single-objective model (17) using the goal programming technique. 


$$
\begin{cases}\min \quad & \sum_{j=1}^{n} S_{j}, \\ & \sum_{r=1}^{s} u_{r} y_{r j}+g_{j}^{\prime}-\sum_{i=1}^{m} v_{i} x_{i j}-f_{j}^{\prime}+S_{j}=0, \quad j=1, \ldots, n \\ & s . t: \sum_{r=1}^{s} u_{r} y_{r j}+g_{j}^{\prime}-\theta_{j}^{*} \sum_{i=1}^{m} v_{i} x_{i j}-\theta_{j}^{*} f_{j}^{\prime} \geq 0, \quad j=1, \ldots, n \\ & f_{j}^{\prime} \geq v_{m+1} \theta_{j}^{*} \lambda_{j} F, \quad j=1, \ldots, n, \\ & g_{j}^{\prime} \geq u_{s+1} \theta_{j}^{*} \mu_{j} G, \quad j=1, \ldots, n, \\ & \sum_{j=1}^{n} f_{j}^{\prime}=v_{m+1} F, \quad j=1, \ldots, n, \\ & \sum_{j=1}^{n} g_{j}^{\prime}=u_{s+1} G, \quad j=1, \ldots, n, \\ & f_{j}^{\prime}, g_{j}^{\prime}, S_{j} \geq 0 u_{r}, v_{i}, u_{s+1}, v_{m+1} \geq \varepsilon, \quad j=1, \ldots, n, \quad r=1, \ldots, s \leq \quad i=1, \ldots, m .\end{cases}
$$

Model (17) is a single-objective linear model that we employ to perform the resource allocation and targeting.

Theorem 2. A feasible solution to the model (17) is always available.

Proof. By setting $v_{m+1}=u_{s+1}=\varepsilon, f_{j}^{\prime}=\varepsilon \lambda_{j} F$, and $g_{j}^{\prime}=\varepsilon \mu_{j} G$, the constraints $4,5,6$, and 7 are satisfied:

$$
\begin{array}{r}
\sum_{j=1}^{n} f_{j}^{\prime}=\sum_{j=1}^{n} \varepsilon \lambda_{j} F=\varepsilon F=v_{m+1} F, \\
\sum_{j=1}^{n} g_{j}^{\prime}=\sum_{j=1}^{n} \varepsilon \mu_{j} G=\varepsilon G=u_{s+1} G, \\
v_{m+1} \theta_{j}^{*} \lambda_{j} F \leq \varepsilon \times 1 \times \lambda_{j} \times F=f_{j}^{\prime}, \\
u_{s+1} \theta_{j}^{*} \mu_{j} G \leq \varepsilon \times 1 \times \mu_{j} \times G=g_{j}^{\prime} .
\end{array}
$$

the second constraint is also satisfied.

By setting $u_{r}=\varepsilon / \beta(\alpha+F)$ and $v_{i}=\varepsilon$, where

$$
\begin{aligned}
& \alpha=\max _{1 \leq j \leq n} \sum_{i=1}^{m} x_{i j}, \\
& \beta=\min _{1 \leq j \leq n} \sum_{r=1}^{s} y_{r j},
\end{aligned}
$$

$$
\begin{aligned}
\theta_{j}^{*} \sum_{i=1}^{m} v_{i} x_{i j}+\theta_{j}^{*} f_{j}^{\prime} & =\theta_{j}^{*}\left(\sum_{i=1}^{m} v_{i} x_{i j}+f_{j}^{\prime}\right) \leq 1 \times\left(\sum_{i=1}^{m} v_{i} x_{i j}+f_{j}^{\prime}\right) \leq \varepsilon \alpha+\varepsilon \lambda_{j} F=\varepsilon\left(\alpha+\lambda_{j} F\right) \\
& =\frac{\varepsilon}{\beta}\left(\alpha+\lambda_{j} F\right) \beta \leq \frac{\varepsilon}{\beta}(\alpha+F) \sum_{r=1}^{s} y_{r j}=\sum_{r=1}^{s} u_{r} y_{r j} \leq \sum_{r=1}^{s} u_{r} y_{r j}+g_{j}^{\prime} .
\end{aligned}
$$

Now, by setting

$S_{j}=-\frac{\varepsilon}{\beta}(\alpha+F) \sum_{r=1}^{s} y_{r j}-\mu_{j} G+\varepsilon \sum_{i=1}^{m} x_{i j}+\lambda_{j} F, \quad j=1, \ldots, n$,

the first constraint is also satisfied.
Theorem 3. Using model (17) for allocation or resource and setting target improve the efficiency.

Proof. Assume that $\left(u_{r}^{*}, v_{i}^{*}, f_{j}^{\prime}{ }^{*}, g_{j}{ }^{*}, v_{m+1}^{*}, u_{s+1}^{*}\right)$ is the best solution for the abovementioned model. Therefore: 


$$
\theta_{j}^{\text {new }}=\frac{\sum_{r=1}^{s} u_{r}^{*} y_{r j}+g_{j}^{\prime} *}{\sum_{i=1}^{m} v_{r}^{*} x_{i j}+f_{j}^{\prime} *} \geq \frac{\theta_{j}^{*} \sum_{r=1}^{s} v_{i}^{*} x_{i j}+\theta_{j}^{*} f_{j}^{\prime} *}{\sum_{i=1}^{m} v_{i}^{*} x_{i j}+f_{j}^{\prime} *}=\theta_{j}^{*} .
$$

\section{Examples}

In this section, we will implement a numerical example and a practical example for the proposed method.

4.1. Numerical Example. Here, for an in-depth comparison, the fixed cost allocation problem of Cook and Kress [2] is used as a numerical example in order to display the application of the resource allocation and target setting approach. This information is given in Table 1 . There are 12 DMUs using 3 inputs to generate 2 outputs. Several studies have used the same example, so it is considered as a key case of resource allocation and target setting without any targets to adjust. Therefore, in this example, $G=0$. The objective is to allocate a common resource $F=100$ (meaning a common cost in this context).

The column 4 of Table 2 provides the efficiency scores of the DMUs calculated using the multiplier CCR model. The efficiency calculations have been carried out using the GAMS software. These calculations merely took a few seconds to finish. The share of each DMU in the input resources $\left(\lambda_{j}\right)$ was calculated using EXCEL, which can be observed in column three of the table. By implementing the proposed model, the cost allocated to each DMU was determined, the results of which are reported in column two of Table 2. And finally, column 5 presents the performance of any DMU following the fixed costs allocation.

The largest contribution in paying the fixed costs is related to DMU 12, which is an efficient DMU and the second DMU with the highest share in the input resources. The lowest contribution is related to DMU 11, which is the least efficient DMU and has a lower share in the inputs compared with other DMUs. Among the efficient DMUs, the lowest contribution in paying the fixed costs is related to DMU5, because it has the lowest share in the input resources. Among the inefficient DMUs, the largest contribution belongs to DMU6, as it has the largest share in the input resources [36-39]. DMUs 1 and 7 have the same share in the inputs resources; however, DMU 7 is more efficient than DMU 1, and as seen in column one of Table 2, DMU 7 has a larger contribution in paying the fixed costs. DMUs 10 and 12 also have the same share in the inputs, but the fixed cost allocated to DMU 12 is greater than that of DMU 10. The reason is that DMU 12 is efficient, while DMU 10 is inefficient. DMUs 9 and 11 also have the same share in the input resources, but there is a large difference in the contribution they make to the fixed costs. The reason is that DMU 9 is an efficient DMU, while DMU 11 has the lowest efficiency score among DMUs. Moreover, the efficiency of all DMUs were improved after allocating the fixed costs, except for DMU 7 whose efficiency did not change at all.

To illustrate some of the important specifications of the proposed approach, the obtained results are compared with
Table 1: A simple example.

\begin{tabular}{lccccc}
\hline DMU & $x_{1}$ & $x_{2}$ & $x_{3}$ & $y_{1}$ & $y_{2}$ \\
\hline 1 & 350 & 39 & 9 & 67 & 751 \\
2 & 298 & 26 & 8 & 73 & 611 \\
3 & 422 & 31 & 7 & 75 & 584 \\
4 & 281 & 16 & 9 & 70 & 665 \\
5 & 301 & 16 & 6 & 75 & 445 \\
6 & 360 & 29 & 17 & 83 & 1070 \\
7 & 540 & 18 & 10 & 72 & 457 \\
8 & 276 & 33 & 5 & 78 & 590 \\
9 & 323 & 25 & 5 & 75 & 1074 \\
10 & 444 & 64 & 6 & 74 & 1072 \\
11 & 323 & 25 & 5 & 25 & 350 \\
12 & 444 & 64 & 6 & 10 & 1199 \\
\hline
\end{tabular}

TABLe 2: Results.

\begin{tabular}{lcccc}
\hline \multirow{2}{*}{ DMU } & Fixed cost & $\lambda_{j}$ & \multicolumn{2}{c}{ CCR efficiency } \\
& & & Before & After \\
\hline 1 & 7.529 & 0.093 & 0.757 & 1.000 \\
2 & 7.985 & 0.074 & 0.923 & 1.000 \\
3 & 7.436 & 0.084 & 0.747 & 1.000 \\
4 & 8.363 & 0.068 & 1.000 & 1.000 \\
5 & 7.647 & 0.058 & 1.000 & 1.000 \\
6 & 10.908 & 0.113 & 0.961 & 1.000 \\
7 & 7.972 & 0.093 & 0.860 & 0.860 \\
8 & 8.203 & 0.068 & 1.000 & 1.000 \\
9 & 10.487 & 0.064 & 1.000 & 1.000 \\
10 & 9.208 & 0.111 & 0.832 & 0.959 \\
11 & 2.140 & 0.064 & 0.333 & 1.000 \\
12 & 12.122 & 0.111 & 1.000 & 1.000 \\
\hline
\end{tabular}

a few other methods as well. The idea of resource allocation based on common weights has been investigated by Beasley [5], Hosseinzadeh et al. [15], Si et al. [40], and Li et al. [10]. All these methods take an approach to resource allocation in which a CSW is determined aiming to achieve maximum efficiency. The results of these comparisons are shown in Table 3.

In the method proposed by Beasley [5], the largest contribution in paying the fixed costs is related to DMU 9. Although DMU 9 is an efficient DMU, it has a small share in the use of input resources as compared with other DMUs. Meanwhile, in our proposed method, the highest payout is related to DMU 12, which is an efficient DMU and is the second DMU with the highest share in the input resources. In all methods, except Li et al. [10], the lowest contribution to fixed costs is related to DMU 11, which has the lowest efficiency score and the lowest share in the input resources. In Li et al. [10], DMU 11 does have the lowest efficiency and the lowest share in the inputs, but the contribution it makes to the fixed costs is greater than that of DMU 9, which is an efficient DMU with a higher share than DMU 11 in the use of input resources. Meanwhile, according to our proposed method, DMU 9 has higher contribution to the fixed costs than DMU 11. In the method presented by Hosseinzadeh 
TABLE 3: Allocations using common weights.

\begin{tabular}{|c|c|c|c|c|c|}
\hline $\mathrm{DMU}$ & Suggested method & Beasly (2003) & Si (2003) & Hosseinzadeh (2013) & $\mathrm{Li}(2017)$ \\
\hline 1 & 7.529 & 6.78 & 7.6475 & 8.199 & 8.7611 \\
\hline 2 & 7.985 & 7.21 & 8.4118 & 7.462 & 7.8689 \\
\hline 3 & 7.436 & 6.83 & 8.6216 & 4.284 & 9.9663 \\
\hline 4 & 8.363 & 8.47 & 8.1091 & 9.301 & 6.9661 \\
\hline 5 & 7.647 & 7.08 & 8.6938 & 4.807 & 7.4589 \\
\hline 6 & 10.908 & 10.06 & 9.5669 & 15.370 & 8.6296 \\
\hline 7 & 7.972 & 5.09 & 8.3333 & 0 & 8.3224 \\
\hline 8 & 8.203 & 7.74 & 9.9628 & 7.339 & 7.7280 \\
\hline 9 & 10.487 & 15.11 & 8.6505 & 16.330 & 7.5359 \\
\hline 10 & 9.208 & 10.08 & 8.3457 & 11.598 & 8.8650 \\
\hline 11 & 2.140 & 1.58 & 2.8032 & 0 & 7.5656 \\
\hline 12 & 12.122 & 13.97 & 11.854 & 15.310 & 10.3291 \\
\hline
\end{tabular}

TABLe 4: An empirical example.

\begin{tabular}{|c|c|c|c|c|c|c|c|c|c|}
\hline $\mathrm{DMU}$ & $x_{1}$ & $x_{2}$ & $x_{3}$ & $y_{1}$ & $y_{2}$ & $f_{j}$ & $g_{j}$ & $\theta$ & $\theta^{\text {new }}$ \\
\hline 1 & 0.150 & 0.713 & 13.3 & 14.478 & 0.232 & 1.709 & 0.567 & 0.602 & 0.845 \\
\hline 2 & 0.170 & 1.071 & 16.9 & 19.502 & 0.340 & 2.347 & 0.805 & 0.607 & 0.886 \\
\hline 3 & 0.235 & 1.224 & 24 & 20.952 & 0.363 & 2.243 & 0.696 & 0.490 & 0.783 \\
\hline 4 & 0.211 & 0.363 & 24 & 13.902 & 0.211 & 1.129 & 0.356 & 0.404 & 0.652 \\
\hline 5 & 0.133 & 0.409 & 15.6 & 15.206 & 0.237 & 1.736 & 0.618 & 0.633 & 0.857 \\
\hline 6 & 0.497 & 5.846 & 18.485 & 81.186 & 1.103 & 12.116 & 4.869 & 1.000 & 1.000 \\
\hline 7 & 0.060 & 0.918 & 56.42 & 81.186 & 1.103 & 10.599 & 4.869 & 1.000 & 1.000 \\
\hline 8 & 0.071 & 1.235 & 12 & 11.441 & 0.199 & 1.253 & 0.325 & 0.418 & 0.712 \\
\hline 9 & 1.500 & 18.12 & 89.51 & 124.072 & 1.858 & 15.209 & 3.308 & 0.424 & 0.668 \\
\hline 10 & 0.120 & 1.821 & 19.8 & 17.425 & 0.274 & 1.838 & 0.454 & 0.404 & 0.663 \\
\hline 11 & 0.120 & 1.915 & 19.8 & 17.425 & 0.274 & 1.833 & 18.790 & 0.440 & 1.000 \\
\hline 12 & 0.050 & 0.874 & 13.1 & 14.342 & 0.177 & 1.691 & 0.465 & 0.396 & 0.725 \\
\hline 13 & 0.370 & 6.918 & 12.5 & 32.491 & 0.648 & 4.400 & 0.929 & 0.565 & 0.731 \\
\hline 14 & 0.440 & 4.432 & 41.9 & 47.653 & 0.636 & 5.611 & 1.389 & 0.390 & 0.691 \\
\hline 15 & 0.431 & 4.504 & 41.1 & 52.63 & 0.741 & 6.461 & 1.744 & 0.543 & 0.744 \\
\hline 16 & 0.110 & 1.241 & 14.4 & 17.493 & 0.243 & 2.126 & 0.605 & 0.571 & 0.768 \\
\hline 17 & 0.053 & 0.450 & 7.6 & 9.512 & 0.067 & 1.175 & 0.294 & 0.667 & 0.668 \\
\hline 18 & 0.345 & 5.892 & 15.5 & 42.469 & 1.002 & 5.950 & 1.897 & 0.552 & 0.990 \\
\hline 19 & 0.128 & 0.973 & 12.6 & 18.987 & 0.243 & 2.465 & 0.816 & 0.737 & 0.847 \\
\hline 20 & 0.055 & 0.444 & 5.6 & 7.546 & 0.153 & 0.952 & 0.367 & 0.658 & 1.000 \\
\hline 21 & 0.057 & 0.508 & 5.7 & 7.595 & 0.123 & 0.951 & 0.308 & 0.619 & 0.862 \\
\hline 22 & 0.098 & 0.370 & 14.1 & 16.906 & 0.233 & 2.085 & 0.794 & 0.778 & 0.898 \\
\hline 23 & 0.104 & 0.395 & 14.6 & 17.264 & 0.263 & 2.120 & 0.837 & 0.746 & 0.933 \\
\hline 24 & 0.206 & 2.680 & 19.6 & 36.43 & 0.601 & 4.913 & 1.709 & 0.710 & 0.935 \\
\hline 25 & 0.067 & 0.781 & 10.5 & 11.581 & 0.120 & 1.363 & 0.337 & 0.547 & 0.663 \\
\hline 26 & 0.100 & 0.872 & 12.1 & 22.207 & 0.248 & 3.019 & 1.122 & 0.920 & 0.887 \\
\hline 27 & 0.0106 & 1.757 & 12.7 & 20.67 & 0.253 & 2.704 & 0.730 & 0.618 & 0.759 \\
\hline
\end{tabular}

et al. [15], DMUs 7 and 11 make no contribution to the fixed costs. DMUs 1 and 7 have the same share of input resources, and DMU 7 has a higher efficiency score than DMU 1. \However, in Hosseinzadeh et al. [15], DMU 7 has no contribution in paying the fixed costs at all, while DMU 1 pays a fixed cost equaling 8.199. In our proposed method, the fixed costs paid by these two DMUs are extremely close to each other, with DMU 7 paying a slightly greater cost. Moreover, in the method provided by Li et al. [10], DMU 3 has the second highest contribution in paying the fixed costs, while it is in the sixth place in terms of the input resources it receives; therefore, it is an inefficient DMU. Furthermore, DMUs 4 and 5 are both efficient, but DMU 4 has a larger share than DMU 5 in the use of input resources, although DMU 4 makes a smaller contribution than DMU 5 in paying the fixed costs. On the other hand, DMU 9, which is an efficient DMU, has a lower contribution in paying the fixed costs.

4.2. An Empirical Example. Kao and Hwang [41] studied the effect of information technology (IT) on the efficiency of banks. We used the same data used in that study, which was related to 27 banks, in order to demonstrate the capability of our proposed method. The inputs included the IT budget $(X 1)$, fixed assets $(X 2)$, and number of employees $(X 3)$, and the outputs were the value of deposits $(Y 1)$ and profits earned (Y2). Columns two to six of Table 4 present a list of data sets. 
First, we calculate the efficiency of the DMUs, as presented in the ninth column of table. As can be observed in this column, two DMUs are efficient. Now, assume that the board of directors in a bank intend to allocate one hundred million dollars of the resources available to them to different branches for equipment updates, and expect that, as a result of this allocation, a total of fifty million dollars be earned by the different branches. Column seven shows the budget allocated to each branch. In the tenth column, the target set for each branch can be observed. As can be seen in these columns, all DMUs are involved in the resource allocation and target setting. In the last column, efficiency scores are calculated after resource allocation and target setting. As can be observed in the table, DMUs 6 and 7 were efficient before resource allocation and target setting, and they are still efficient after the allocation. Moreover, DMUs 11 and 20, which were inefficient before allocation, have become efficient after the allocation. According to the last two columns in the table, the efficiency of all DMUs has either increased after resource allocation or remained the same as before allocation. The number of efficient DMUs has increased from 2 to 4 . The lowest efficiency score belongs to DMU 14. The highest amount of allocated resources belongs to DMU 9, and as can be seen, it has the highest amount of inputs and outputs among all DMUs. After DMU 9, most of the allocated resources are related to DMUs 6 and 7 , respectively, both of which are efficient. And they remain efficient after allocating resources and setting targets.

The lowest amount of allocated resources pertains to DMU 21, which has a lower amount of inputs compared with other DMUs. And then the lowest allocation belongs to DMUs 20 and 4, respectively, both of which are inefficient. DMU 11 has had the highest amount of changes in efficiency, the reason for which can be related to the target set for the DMU. DMUs 4 and 10 had the same efficiency before allocation, but their efficiency scores changed after the resource allocation and target setting. The reason for this could be related to their inputs, as DMU 10 has a lower ratio of inputs to overall inputs than DMU 4.

Based on the efficiency scores after resource allocation and target setting, the lowest amount of changes in efficiency is related to DMU 17, which is an inefficient DMU and the least targeting has been done for it. Moreover, its received resources are less than other DMUs. The reason for this is that the lowest target was set for this DMU, and it also has received a lower amount of resources than other DMUs, which was itself due to the DMU having a lower amount of inputs and outputs compared with other DMUs. The highest amount of changes is observed in DMU 11, as the highest target was set for this DMU.

\section{Conclusion}

In today's competitive world, companies and organizations employ scientific methods to make better use of resources and achieve the intended goals. Many researchers have considered fixed resource allocation and targeting in data envelopment analysis science [42-45]. Resource allocation is done for different purposes. Some organizations prefer to allocate resources to prevent changes in the efficiency of
DMUs [46-49]. However, the objective of other organizations is to increase efficiency. In this paper, resource allocation and targeting are performed to increase efficiency using a CSW.

In all models previously presented for allocating resources and setting targets, the involvement of all DMUs in the resource allocation and target setting was neglected [50-53]. We proposed a model that involves all DMUs in the procedure.

The efficiency of the DMUs is improved following the resource allocation and target setting. Here, a lower bound is considered for the allocation of resources and target setting based on the efficiency of each DMU, the share it has in the use of input resources relative to other DMUs, and the outputs it produces relative to other DMUs. This method was compared with other methods provided by common weights by presenting a numerical example. An applied example was also employed with this method.

For future research, an upper bound can also be determined. In future research, resource allocation can be performed using common weights with undesired inputs and outputs [54-57]. The resource allocation using goal programming along with fuzzy data can be one of the interesting topics for future researches [58-61].

\section{Data Availability}

Data are available on request from the corresponding author.

\section{Conflicts of Interest}

The authors declare that they have no conflicts of interest.

\section{References}

[1] A. Charnes, W. W. Cooper, and E. Rhodes, "Measuring the efficiency of decision making units," European Journal of Operational Research, vol. 2, no. 6, pp. 429-444, 1978.

[2] W. D. Cook and M. Kress, "Characterizing an equitable allocation of shared costs: a DEA approach," European Journal of Operational Research, vol. 119, no. 3, pp. 652-661, 1999.

[3] W. D. Cook and J. Zhu, "Allocation of Shared Costs Among Decision Making Units: A Dea approach," Computers \& Operations Research, vol. 32, no. 8, pp. 2171-2178, 2005.

[4] R. Lin, "Allocating fixed costs or resources and setting targets via data envelopment analysis," Applied Mathematics and Computation, vol. 217, no. 13, pp. 6349-6358, 2011.

[5] J. E. Beasley, "Allocating fixed costs and resources via data envelopment analysis," European Journal of Operational Research, vol. 147, no. 1, pp. 198-216, 2003.

[6] A. Amirteimoori and S. Kordrostami, "Allocating fixed costs and target setting: a dea-based approach," Applied Mathematics and Computation, vol. 171, no. 1, pp. 136-151, 2005.

[7] G. R. Jahanshahloo, J. Sadeghi, and M. Khodabakhshi, "Using dea based on the efficiency invariance and common set of weights principles," Mathematical Methods of Operations Research, vol. 85, pp. 223-240, 2017.

[8] F. Li, J. Song, A. Dolgui, and L. Liang, "Using common weights and efficiency invariance principles for resource allocation and target setting," International Journal of Production Research, vol. 55, no. 17, pp. 4982-4997, 2017. 
[9] G. R. Jahanshahloo, F. Hosseinzadeh Lotfi, N. Shoja, and M. Sanei, "An alternative approach for equitable allocation of shared costs by using DEA," Applied Mathematics and Computation, vol. 153, no. 1, pp. 267-274, 2004.

[10] Y. Li, F. Yang, L. Liang, and Z. Hua, "Allocating the fixed cost as a complement of other cost inputs: a DEA approach," European Journal of Operational Research, vol. 197, no. 1, pp. 389-401, 2009.

[11] J. Du, W. D. Cook, L. Liang, and J. Zhu, "Fixed cost and resource allocation based on DEA cross-efficiency," European Journal of Operational Research, vol. 235, no. 1, pp. 206-214, 2014.

[12] F. H. Lotfi, M. Rostamy-Malkhalifeh, M. R. Mozaffari, M. H. Behzadi, and M. Ghasemi, "Fair allocation of fixed costs in data envelopment analysis," Advances in Intelligent Systems and Computing, vol. 1301, pp. 399-405, 2021.

[13] S. Asghariniya, H. Z. Rezai, and S. Mehrabian, "Resource allocation: a common set of weights model," Numerical Algebra Control and Optimization, vol. 10, pp. 257-273, 2020.

[14] A. Amirteimoori and M. Mohaghegh Tabar, "Resource allocation and target setting in data envelopment analysis," Expert Systems with Applications, vol. 37, pp. 3036-3039, 2010.

[15] F. Hosseinzadeh Lotfi, A. Hatami-Marbini, P. J. Agrell, N. Aghayi, and K. Gholami, "Allocating fixed resources and setting targets using a common-weights DEA approach," Computers \& Industrial Engineering, vol. 64, pp. 631-640, 2013.

[16] R. G. Thompson, F. D. Singleton, R. M. Thrall, and B. A. Smith, Comparative site evaluations for locating a highenergy physics lab in Texas, vol. 16, pp. 35-49, 1986.

[17] R. Thompson, F. D. Singleton, R. M. Thrall, and B. A. Smith, "Systems task force comparative site evaluations for the Texas," Interfaces, vol. 16, pp. 35-49, 1985.

[18] W. D. Cook, Y. Roll, and A. Kazakov, "A dea model for measuring the relative efficiency of highway Maintenance Patrols," INFOR: Information Systems and Operational Research, vol. 28, pp. 113-124, 1990.

[19] A. Charnes, W. Cooper, Q. L. Wei, and Z. Huang, "Cone ratio data envelopment analysis and multi-objective programming," International Journal of Systems Science, vol. 20, pp. 1099-1118, 1989.

[20] Y. Roll, W. D. Cook, and B. Golany, "Controlling factor weights in data envelopment analysis," IIE Transactions, vol. 23, pp. 2-9, 1991.

[21] F. Hosseinzadeh Lotfi, G. R. Jahanshahloo, and A. Memariani, "A method for finding common set of weights by multiple objective programming in data envelopment analysis," Southwest Journal of Pure and Applied Mathematics, vol. 1, pp. 44-54, 2000.

[22] G. R. Jahanshahloo, A. Memariani, F. Hosseinzadeh Lotfi, and H. R. Rezai, "A note on some of DEA models and finding efficiency and complete ranking using common set of weights," Applied Mathematics and Computation, vol. 166, pp. 265-281, 2005.

[23] W. D. Cook and J. Zhu, "Within group common weights in DEA: an analysis of power plant efficiency," European Journal of Operational Research, vol. 178, pp. 207-216, 2007.

[24] A. Davoodi and H. Z. Rezai, "Common set of weights in data envelopment analysis: a linear programming problem," Central European Journal of Operations Research, vol. 20, pp. 355-365, 2012.

[25] M. Kachouei, A. Ebrahimnejad, and H. Bagherzadeh-Valami, "A common-weights approach for efficiency evaluation in fuzzy data envelopment analysis with undesirable outputs: application in banking industry," Journal of Intelligent and Fuzzy Systems, vol. 39, pp. 7705-7722, 2020.

[26] J. Zhang, C. Shen, H. Su, M. T. Arafin, and G. Qu, "Voltage over-scaling-based lightweight Authentication for IoT security," IEEE Transactions on Computers, p. 1, 2021.

[27] B. Li, R. Liang, W. Zhou, H. Yin, H. Gao, and K. Cai, "LBS meets blockchain:an efficient method with security preserving trust in SAGIN," IEEE Internet of Things Journal, vol. 1, 2021.

[28] L. Hua, H. Zhu, K. Shi, S. Zhong, Y. Tang, and Y. Liu, "Novel finite-time reliable control design for memristor-based inertial neural networks with mixed time-varying delays," IEEE transactions on circuits and systems. I, Regular papers, vol. 68, no. 4, pp. 1599-1609, 2021.

[29] Z. Wu, J. Cao, Y. Wang, Y. Wang, L. Zhang, and J. Wu, "hPSD: a hybrid PU-Learning-Based spammer detection model for product reviews," IEEE Transactions on Cybernetics, vol. 50, no. 4, pp. 1595-1606, 2020.

[30] N. Xiao, R. Xinyi, Z. Xiong et al., "A diversity-based selfish node detection algorithm for socially aware networking," Journal of signal processing systems, vol. 93, pp. 811-825, 2021.

[31] B. Li, G. Xiao, R. Lu, R. Deng, and H. Bao, "On feasibility and limitations of detecting false data injection attacks on power grid state estimation using D-FACTS devices," IEEE Transactions on Industrial Informatics, vol. 16, no. 2, pp. 854-864, 2020.

[32] M. Tamiz, D. Jones, and C. Romero, "Goal programming for decision making: an overview of the current state-of-the-art," European Journal of Operational Research, vol. 111, pp. 569581, 1998.

[33] J. Feng, Z. Liu, and L. Feng, "Identifying opportunities for sustainable business models in manufacturing: application of patent analysis and generative topographic mapping," Sustainable Production and Consumption, vol. 27, pp. 509-522, 2021.

[34] X. Chen, T. Wang, R. Ying, and Z. Cao, “A fault diagnosis method considering meteorological factors for transmission networks based on P systems," Entropy, vol. 23, no. 8, 2021.

[35] T. Wang, W. Liu, J. Zhao, X. Guo, and V. Terzija, "A rough set-based bio-inspired fault diagnosis method for electrical substations," International Journal of Electrical Power \& Energy Systems, vol. 119, Article ID 105961, 2020.

[36] T. Wang, X. Wei, J. Wang et al., "A weighted corrective fuzzy reasoning spiking neural $P$ system for fault diagnosis in power systems with variable topologies," Engineering Applications of Artificial Intelligence, vol. 92, Article ID 103680, 2020.

[37] Z. Huang, T. Wang, W. Liu et al., "A fault analysis method for three-phase induction motors based on spiking neural P systems," Complexity, vol. 2021, Article ID 2087027, 19 pages, 2021.

[38] C. Liu, X. Gao, D. Chi, Y. He, M. Liang, and H. Wang, "Online chatter detection in milling using fast kurtogram and frequency band power," European Journal of Mechanics, A, Solids, vol. 90, Article ID 104341, 2021.

[39] Y. L. X. L. Yang Yanga, "Anthropomorphism and customers'willingness to use artificial intelligence service agents," Journal of Hospitality Marketing \& Management, 2021.

[40] P. Wang and Y. S. E. M. A. Liu, "Secure and efficient message authentication protocol for VANETs," IEEE systems journal, vol. 15, no. 1, pp. 846-855, 2021.

[41] S. Lv and Y. Liu, "PLVA: privacy-preserving and lightweight V2I authentication protocol," IEEE Transactions on Intelligent Transportation Systems, pp. 1-7, 2021. 
[42] J. Chen, Y. Liu, Y. Xiang, and K. Sood, "RPPTD: Robust privacy-preserving truth discovery scheme," IEEE systems journal, pp. 1-8, 2021.

[43] Y. Feng, B. Zhang, Y. Liu et al., "200-225-GHz manifoldcoupled multiplexer utilizing metal wave guides," IEEE Transactions on Microwave Theory and Techniques, vol. 69, no. 12, pp. 5327-5333, 2021.

[44] X. Cai, J. Wang, S. Zhong, K. Shi, and Y. Tang, "Fuzzy quantized sampled-data control for extended dissipative analysis of T-S fuzzy system and its application to WPGSs," JOURNAL OF THE FRANKLIN INSTITUTE-ENGINEERING AND APPLIED MATHEMATICS, vol. 2, no. 358, pp. 1350-1375, 2021.

[45] X. Cai, S. Zhong, J. Wang, and K. Shi, "Robust Ho control for uncertain delayed T-S fuzzy systems with stochastic packet dropouts," Applied Mathematics and Computation, vol. 385, Article ID 125432, 2020.

[46] X. Cai, K. Shi, S. Zhong, J. Wang, and Y. Tang, "Dissipative analysis for high speed train systems via looped-functional and relaxed condition methods," Applied Mathematical Modelling, vol. 96, pp. 570-583, 2021.

[47] X. Cai, K. Shi, S. Zhong, and X. Pang, "Dissipative sampleddata control for high-speed train systems with quantized measurements," IEEE Transactions on Intelligent Transportation Systems, vol. 99, pp. 1-12, 2021.

[48] G. Sun, Y. Cong, Q. Wang, B. Zhong, and Y. Fu, "Representative task self-selection for flexible clustered lifelong learning," IEEE transaction on neural networks and learning systems, pp. 1-15, 2020.

[49] H. Yi, "Secure social internet of things based on post-quantum blockchain," IEEE transactions on network science and engineering, vol. 1, 2021.

[50] Y. Ma and D. Bennett, "The relationship between higher education students' perceived employability, academic engagement and stress among students in China," Education \& training (London), vol. 63, no. 5, pp. 744-762, 2021.

[51] A. Roghani, "The relationship between macro-socioeconomics determinants and COVID-19 vaccine distribution," AIMS Public Health, vol. 8, no. 4, pp. 655-664, 2021.

[52] X. Si, L. Liang, G. Jia, L. Yang, H. Wu, and Y. Li, "Proportional sharing and DEA in allocating the fixed cost," Applied Mathematics and Computation, vol. 219, pp. 6580-6590, 2013.

[53] C. Kao and S. N. Hwang, "Efficiency measurement for network systems: IT impact on firm performance," Decision Support Systems, vol. 48, pp. 437-446, 2010.

[54] M. Sharma, H. Pham, and V. Singh, "Modeling and analysis of leftover issues and release time planning in multi-release open source software using entropy based measure," Computer Systems Science and Engineering, vol. 34, no. 1, pp. 33-46, 2019.

[55] S. Baek, J. Jeon, B. Jeong, and Y. S. Jeong, "Two-stage hybrid malware detection using deep learning," Human-centric Computing and Information Sciences, vol. 11, no. 27, 2021.

[56] Y. Chen, K. Li, W. Yang, G. Xiao, X. Xie, and T. Li, "Performance-aware model for sparse matrix-matrix multiplication on the sunway TaihuLight supercomputer," IEEE Transactions on Parallel and Distributed Systems, vol. 30, no. 4, pp. 923-938, 2019.

[57] A. Ebrahimnejad and N. Amani, "Fuzzy data envelopment analysis in the presence of undesirable outputs with ideal points," Complex Intell. Syst.vol. 7, pp. 379-400, 2021.

[58] C. Chen, K. Li, A. Ouyang, and K. Li, "FlinkCL: an OpenCLbased in-memory computing architecture on heterogeneous CPU-gpu clusters for big data," IEEE Transactions on Computers, vol. 67, no. 12, pp. 1765-1779, 2018.
[59] J. Mei, K. Li, T. Zhao, Q. Li, and K. Li, "Profit maximization for cloud brokers in cloud computing," IEEE Transactions on Parallel and Distributed Systems, vol. 30, no. 1, pp. 190-203, 2019.

[60] M. Duan, K. Li, K. Li, and T. Qi, "A novel multi-task tensor correlation neural network for facial attribute prediction," ACM Trans. Intell. Syst. Technol, vol. 12, no. 1, pp. 1-3, 2021.

[61] D. Di Caprio, A. Ebrahimnejad, and M. Ghiyasi, "Integrating fuzzy goal programming and data envelopment analysis to incorporate preferred decision-maker targets in efficiency measurement," Decisions in Economics and Finance, vol. 43, pp. 673-690, 2020. 